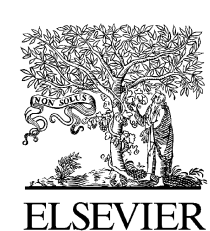

\title{
EDITORIAL
}

\section{Editor's Introduction to the Journal}

\section{Steve E. Abraham*}

\author{
Editor-in-Chief of the Journal
}

I listened to a lecture by the 14th Dalai Lama in which he said, "From my own limited experience I have found that the greatest degree of inner tranquility comes from the development of love and compassion." ${ }^{\text {I began to }}$ think about how much medicine has changed and why the Journal of the American College of Certified Wound Specialists is necessary. For a professional, treating a patient with kindness and compassion has a distinct and clear meaning that may not seem apparent. In my perspective, showing kindness and compassion to a patient means following accepted evidence-based protocols so that the patient has the best chance of a positive outcome from the encounter.

The medical expert who relies only on experience and opinion has been demoted. Genuine compassion and kindness can happen when the medical practitioner becomes a true professional, not just a dabbler in medicine. Today, the image of the medical professional is one whose decisions are based on firm scientific principles that have been tested and developed into protocols, not one who relies on subjective experience and opinion. The medical professional uses evidence-based medicine (EBM). A professional journal on wound care needs to be a peerreviewed journal that bases its publication on EBM. We can demonstrate compassion for our patients by understanding the scientific basis for our decisions and providing treatment using protocols based on EBM. This is the purpose of this journal. The expert is expected to reference all decisions on patient care, and the sources of this information are scrutinized for scientific validity and accuracy. $^{2}$

\footnotetext{
*Corresponding author.

E-mail address: ejwounds@aol.com
}

The modern concept of EBM was developed by a group of Canadian internists at McMaster University, in Hamilton, Ontario. Their work led to a series of articles published in the Journal of the American Medical Association titled "The User's Guide to the Medical Literature." 3 The term EBM was introduced into the literature by Guyat et al in $1992 .^{4}$ EBM is a system used to uniformly apply the standards of the scientific method to medicine. According to the Centre for Evidence-Based Medicine, "EBM is the conscientious, explicit and judicious use of current best evidence in making decisions about the care of individual patients." 3

A scientist starts with a hypothesis, which is essentially an assumption. The scientist needs to assess the validity of the hypothesis and designs experiments, collects data, and analyzes the data to see whether they support the hypothesis. Based on the data, the hypothesis can be accepted or rejected or the results labeled as inconclusive. More-refined experiments can be devised to support or evaluate the hypothesis again. Scientists must then communicate their experiments and findings to their peers, who read and evaluate the hypothesis, the experiments, the data, and the data evaluation process. This happens before the scientist's manuscripts are published. After publication, other scientists must replicate the original experiments to verify the original blinded article's research. These second results are published, and they verify, challenge, or expand on the original claims. ${ }^{5}$

There is no secrecy to EBM; it must be transparent. Knowledge is not spread by nonreplicable methods or from revelation, witnesses, or testimonials of people whose lives were allegedly affected. A professional is one who uses both individual clinical experience and best available external evidence and who understands that neither alone is enough. ${ }^{6}$

I would like to thank a few individuals who have significantly contributed to the creation of this journal. 
We could not have come so far with this journal, in such a short amount of time, without your dedication.

First, the Elsevier staff who have guided us through the initial setup, creation of the Elsevier Editorial System (EES) for manuscript submission, online peer review process, copy editing, and finally publishing. Chris Baumle has guided us from the beginning. Scott Shookhoff taught us how to use the EES online site for submissions and reviews. Chris and Scott have been kind and patient throughout the learning process. Anyone who has tried to teach someone computer programs knows how frustrating that can be.

Several reviewers have made a significant impact on our journal, as well. The peer review process needs to have high professional standards that cannot be compromised. Dr. Jane Fore has been instrumental in keeping us on the right path of professionalism and has been an inspiration to the editors. Dr. Wayne Stewart has made sure our review process is strict and of the highest level. Dr. Richard Simman's helpful critiques have kept us from making compromises in our standards. Luther Kloth points out our inaccuracies in a kind way. Thank you to the entire editorial review board for your quality reviews.

The staff members at the American College of Certified Wound Specialists office have been patient and ready at a moment's notice to do whatever is necessary to keep the momentum rolling.

\section{Acknowledgment}

A special thanks to Kari Bechert, assistant editor-in-chief. Without Kari's enthusiasm, work ethic, and persistence, together with her knowledge, intelligence, and common sense, this journal would have been difficult to produce.

And finally, thanks to the spouses of both Kari and I, for their patience and help through this process.

\section{References}

1. Gyatso, T (H.H. the 14th Dalai Lama). Compassion and the individual. Foundation for the Preservation of the Mayahana Tradition Web site. Available at: http://www.fpmt.org/teachings/hhdl/compassionind.asp. Accessed November 21, 2008.

2. Henderson JA. Fear, Faith, Fact, Fantasy. Boone, NC: Parkway; 2003.

3. Waldman, MH. Evidence-based health care: the art of translating clinical research into patient care. Newsletter of the American College of Foot \& Ankle Orthopedics \& Medicine. 2006; winter:9-12.

4. Center for Evidence-Based Medicine. Glossary of EBM terms. Available at: http://www.cebm.utoronto.ca/glossary/. Accessed November 21, 2008.

5. Elsevier Editorial System: A Guide for Journal Editors and Editorial Offices [computer program]. Version 2.

6. Sackett, DL, Rosenberg, WM, Gray, JM, Haynes, RB, Richardson, WS. Evidence-based medicine: what it is and what it isn't. BMJ. 1996;312: 71-72. Available at: http://www.bmj.com/cgi/content/full/312/7023/71. Accessed November 21, 2008. 